FORMATION Formation emploi

Revue française de sciences sociales

94 | avril-juin 2006

Immigration et marché du travail

\title{
Toutes choses inégales d'ailleurs : six études sur les discriminations
}

\section{François Héran}

\section{(2) OpenEdition}

1 Journals

Édition électronique

URL : http://journals.openedition.org/formationemploi/2167

DOI : 10.4000/formationemploi.2167

ISSN : 2107-0946

Éditeur

La Documentation française

Édition imprimée

Date de publication : 1 avril 2006

Pagination : 5-10

ISSN : 0759-6340

\section{Référence électronique}

François Héran, «Toutes choses inégales d'ailleurs : six études sur les discriminations », Formation emploi [En ligne], 94 | avril-juin 2006, mis en ligne le 01 décembre 2009, consulté le 30 octobre 2020. URL : http://journals.openedition.org/formationemploi/2167 ; DOI : https://doi.org/10.4000/

formationemploi.2167

(c) Tous droits réservés 
accent, patronyme d'allure étrangère, origine étrangère...), la justice peut lui demander de rendre des comptes à ce sujet : le contrôleur du droit d'accès at-il pris la mesure des inégalités engendrées par son activité ? Quelles dispositions a-t-il prises pour les prévenir ou les corriger? La notion de « discrimination objective » ne consiste donc pas à objectiver le problème en le dépersonnalisant mais, tout au contraire, à repersonnaliser une situation qui pouvait sembler impersonnelle, en cherchant un responsable par omission.

Les six études reunies ici offrent autant de variations sur ces thèmes. Ilke Adam montre à propos du cas belge à quel point la pratique du « test de situation » (ou testing), popularisée par une enquête du Bureau international du travail (BIT), est parvenue à dessiller les yeux de l'opinion publique, qui y a reconnu très rapidement une preuve indubitable des comportements discriminatoires. En fait, le test de situation représente en psychologie du comportement l'équivalent exact de l'analyse " toutes choses égales par d'ailleurs » en statistique sociale. Dans les deux cas, il s'agit d'étudier la variation sur fond d'invariant. La technique de la régression logistique pratiquée à cet effet par les statisticiens consiste bien à égaliser toutes les conditions de la comparaison pour mesurer séparément l'incidence de chaque facteur sur le comportement. Dans le testing comme dans la régression logistique, l'effet d'une variable s'apprécie en neutralisant toutes les autres différences (sexe, âge, instruction, etc.). Les situations que l'on compare ne diffèrent plus alors que par un seul trait: un signe perceptible d'appartenance à un groupe (apparence physique, patronyme, pays de naissance...). Si cette appartenance suffit à obérer les chances d'accès de façon significative, indépendamment des autres atouts ou handicaps sociaux de l'individu, alors le doute n'est plus permis : il y a inégalité de traitement et discrimination.

Le parallèle s'arrête là. Autant la régression logistique est difficile à présenter au grand public, autant le test en situation est susceptible de le toucher. Du côté de l'analyse statistique, l'égalisation des conditions de la comparaison est le fruit d'une expérience de pensée pour le moins abstraite, tandis que du côté du testing, elle prend la forme d'une expérience concrète : on soumet à la même épreuve deux postulants semblables à tous égards - à un détail visible près. S'il apparaît qu'ils sont traités différemment par le même acteur, il ne fera de doute pour personne que l'inégalité de traitement tient à la prise en compte de ce détail. Le pouvoir probatoire du testing semble renforcé par le fait qu'il s'apparente à la narration très simple des contes, dont la structure est souvent celle d'une série d'épreuves infligées au même héros ou d'une série de prétendants passant la même épreuve : chacun peut s'identifier au héros de l'histoire - et sans doute aussi se mettre à la place inavouable de l'anti-héros, le discriminateur. Rien de tel dans l'analyse statistique multivariée, qui ne livre que des vérités probabilistes ou, comme disent encore les statisticiens, des «associations préférentielles entre variables ». Enfin, le testing donne l'impression qu'il suffit, pour conclure à une injustice flagrante, de comparer à moindres frais le sort de deux postulants, tandis que l'analyse statistique requiert pour sa part de gros échantillons pour prendre en compte la diversité des personnes et espérer atteindre un résultat significatif.

Bien entendu, ce contraste est fortement exagéré : la psychologie expérimentale a beau travailler sur des effectifs plus réduits que la statistique sociale (quelques dizaines de cas au lieu de quelques milliers), elle n'en doit pas moins se soumettre elle aussi au test de la significativité des résultats, ce qui lui confère une dimension statistique, même s'il s'agit d'une statistique reposant davantage sur des «plans d'expérience » que sur des échantillons représentatifs. Par ailleurs, les deux méthodes se sont singulièrement rapprochées avec l'entrée en scène des testings de deuxième génération, qui ne consistent plus à envoyer quelques individus en chair et en os se présenter devant un guichet ou à l'entrée d'une boîte de nuit, mais à adresser à un échantillon d'employeurs un grand nombre de demandes d'emploi (jusqu'à quelques milliers), présentant quelques profils de candidats dont on fait varier les caractéristiques.

Ilke Adam s'interroge sur les conditions qui peuvent favoriser le développement des études de discrimination et leur diffusion. Ce qu'elle mentionne pour la Belgique vaut pour la France et d'autres pays : la dénonciation des discriminations à l'embauche subies par les immigrés a davantage le vent en poupe quand la conjoncture économique devient favorable (comme ce fut le cas à la fin des années 1990) et que les employeurs commencent à se préoccuper des pénuries de main-d'œuvre. Un facteur d'une tout autre nature est la pression des institutions euro- 
péennes. Pour ne prendre que l'exemple de la France, la création récente de la Haute autorité de lutte contre les discriminations et pour l'égalité (HALDE) n'a pas surgi du débat public interne; nous la devons aux directives européennes de l'an 2000, elles-mêmes liées à l'adoption du traité d'Amsterdam.

Lauteur invoque aussi la division du travail entre le monde de la recherche et le monde politique. Si les travaux des chercheurs ne sont pas relayés et valorisés par les politiques (ou, du moins, une fraction suffisamment active des politiques), leur écho reste faible. Reste à savoir ce que l'on entend par politiques. En France, les services d'études et de statistiques des administrations centrales parviennent à garder une certaine autonomie par rapport au personnel politique qui est censé les utiliser. L'une des raisons en est que la production statistique est largement dans les mains des experts diplômés des écoles de l'Insee, qui contribuent, au sein de ces directions, à protéger tant bien que mal les analyses statistiques des interventions politiques. Les publications des administrations centrales restent toutefois confidentielles, malgré le recours à des supports de petit format (quatre pages ou six pages). Seul l'Insee, fort de ses effectifs et de sa couverture territoriale, parvient à publier ses analyses à un rythme soutenu et avec un large écho médiatique.

L'immigration constitue toutefois un cas très particulier: la dispersion des sources sur les flux et les stocks, le soupçon régulièrement entretenu par certains groupes de pression sur la partialité des estimations chiffrées, ont conduit les politiques à dénoncer la cacophonie des données, mais avec l'effet paradoxal d'ajouter de nouveaux rapports à ceux qui existaient déjà. Il existe à l'heure actuelle trois publications : le rapport annuel de la Direction de la population et des migrations (ministère de l'Emploi), le rapport annuel de l'Observatoire des statistiques de l'immigration et de l'intégration (Haut conseil à l'intégration, dépendant de la présidence de la République) et le rapport annuel du ministère de l'Intérieur au Parlement (préparé par le Comité interministériel de contrôle de l'immigration). Ces données s'ajoutent aux estimations publiées chaque année par l'Insee dans son bilan démographique et complétées par l'Ined.

Le contraste est flagrant avec les travaux sur la discrimination, qui ne font l'objet d'aucun suivi statistique d'ensemble. Les données administratives ne permet- tent pas de repérer les populations-cibles, que ce soit dans la sphère des relations de travail ou dans la sphère judiciaire. En tout état de cause, les statistiques du ministère de la Justice, après avoir été historiquement en pointe, sont revenues au stade d'une simple statistique de gestion, en raison de la surcharge croissante des greffes qui doivent les entretenir. Une issue possible est en train de s'ouvrir, toutefois, avec l'enrichissement du questionnaire de l'enquête Emploi depuis 2005 : de nouvelles questions sur les pays de naissance des parents vont permettre de repérer les enfants d'immigrés et d'étudier chaque année les chances d'accès à la formation, à l'emploi et à la promotion, en fonction des origines nationales des personnes. Là encore, ces nouveautés résultent davantage des injonctions d'Eurostat (agence statistique européenne) à l'échelle européenne que d'une demande précise du corps politique national.

Faute de pouvoir suivre la conjoncture de l'intégration et de la discrimination avec des données régulières, les statisticiens de l'Insee et les chercheurs de l'Ined sont obligés d'investir, à intervalles espacés de plus de dix ans, dans des enquêtes transversales particulièrement lourdes : en 1992, l'enquête MGIS (Mobilité géographique et insertion sociale des migrants); l'enquête $\mathrm{TeO}$ (Trajectoires et origines des migrants et de leurs descendants), en cours de préparation, sans oublier les apports partiels de l'enquête Famille associée au recensement de 1999 ou de l'enquête Histoires de vie / Construction des identités de 2003.

Or, l'article d'Alain Frickey et Jean-Luc Primon rappelle fort opportunément qu'un autre type de source est disponible, de type longitudinal celui-là, à savoir les enquêtes Générations du Cereq, en particulier celle qui a suivi, en 2001, les élèves ou étudiants sortis du système éducatif en 1998, au moyen d'une grille de parcours professionnel mois par mois. Détail important, le questionnaire permet d'identifier les enfants d'immigrés grâce à des questions sur le pays de naissance des deux parents. Centré sur les étudiants ayant quitté l'Université sans diplôme validé, le travail des deux sociologues débouche sur une analyse «toutes choses égales par ailleurs » qui établit clairement que les étudiants d'origine maghrébine, et plus particulièrement les femmes, sont systématiquement désavantagés par rapport aux enfants de migrants venus de l'Europe du Sud et aux étudiants d'origine française, pour toute une série d'indica- 
teurs : la rapidité de l'entrée sur le marché du travail, la durée cumulée du chômage, la probabilité d'obtenir un emploi aidé ou de travailler à temps partiel. Ces écarts sont aggravés par le fait de résider en province ou d'avoir une mère inactive (situation majoritaire dans les familles d'origine maghrébine). De façon générale, le sexe et l'origine nationale cumulent leurs effets. Les auteurs relèvent aussi que les «Emplois jeunes » ont particulièrement bénéficié aux jeunes femmes issues de familles maghrébines, en les maintenant en emploi de façon transitoire, avec cet effet secondaire pour le moins artificiel de relever la part des "professions intermédiaires» dans la structure des emplois occupés.

On remarquera la prudence des auteurs sur la question de la discrimination. Ils parlent d'une catégorie « doublement pénalisée » à raison de son sexe et de ses origines, en mettant davantage en avant les handicaps sociaux hérités de la génération précédente (comme le faible niveau d'instruction des mères et leur faible taux d'activité) que les comportements discriminatoires des employeurs. Il est vrai que la démonstration d'une discrimination est plus difficile à administrer quand il est question de jeunes sans diplôme. En revanche, elle est immédiatement sensible quand on peut démontrer que deux titulaires d'un même diplôme n'en obtiennent pas le même rendement sur le marché du travail, du simple fait qu'ils n'ont pas la même origine. En l'absence de diplôme, l'interprétation des inégalités est plus délicate. On peut imaginer que les jeunes d'origine française ou européenne éprouvent relativement moins de difficultés que les jeunes originaires du Maghreb parce qu'ils ont plus d'atouts hérités, comme le capital social qui permet aux enfants de Portugais ou d'Espagnols de s'orienter plus aisément vers les petites entreprises. L'hypothèse de comportements discriminatoires qui cibleraient les jeunes Maghrébines sans diplôme, davantage que leurs homologues masculins, demanderait à être confirmée par d'autres recherches.

C'est ici que la démonstration apportée par Rosita Fibbi sur le cas de la Suisse est particulièrement éclairante. La méthode est celle du test en situation réelle, appliquée par l'Organisation internationale du travail (OIT). Elle consiste à envoyer des candidatures à pour des offres d'emploi parues dans la presse. Les résultats sont comparés pour deux candidats fictifs ne différant que par le pays d'origine (par exemple
Pierre Droz versus Ekim Belezi). Un premier taux de discrimination mesure la proportion d'envois jumeaux qui se soldent par une proposition d'entretien pour le candidat suisse et non pour le candidat issu de l'immigration. Un second taux (dit « taux maximal ») y ajoute les cas où l'employeur ne prend contact avec le candidat de l'immigration qu'après le refus de la même offre par le candidat suisse. Les résultats pour ce dernier taux sont impressionnants : de $42 \%$ à $70 \%$, selon les groupes, le record s'observant en Suisse germanophone vis-à-vis des Yougoslaves. Ces taux sont très supérieurs aux taux observés par l'OIT dans d'autres pays européens.

Rosita Fibbi suggère plusieurs explications ou, à tout le moins, des éléments de contexte: une politique migratoire qui considère de longue date que les migrants sont des invités temporaires et leur impose une rotation élevée, une politique de la citoyenneté qui multiplie les obstacles à la naturalisation (on ne peut devenir Suisse qu'en étant littéralement adopté par la commune, le canton et la Fédération), et des discours xénophobes récurrents dans le débat public. Elle reconnaît cependant qu'une fois établi le constat de la discrimination par le testing jumelé, il reste à enquêter sur les motivations des employeurs, qui ne peuvent être étudiées que moyennant des entretiens approfondis et des observations suivies, en tenant compte des relations perçues ou vécues entre tous les acteurs : l'employeur, le collectif de travail, l'environnement immédiat de l'entreprise, les autorités locales.

L'enquête de Frédéric Charles se situe encore dans le registre quantitatif mais prend un tour plus monographique en interrogeant un millier d'élèves de seconde année de l'IUFM (Institut universitaire de formation des maîtres) de Créteil. Sur ce nombre, $18 \%$ ont au moins un parent immigré et se signalent par des caractéristiques singulières : un soutien parental très fort, malgré le faible niveau d'instruction des mères, des attaches préalables avec le monde éducatif et, plus largement, avec la fonction publique.

Bien entendu, l'étude interne d'une population qui est parvenue à entrer dans une institution donnée ne peut livrer les mêmes résultats qu'une analyse des chances d'accès à l'institution, qui supposerait de réintégrer les populations environnantes dans l'analyse. Cependant, les quelques indications données par l'auteur semblent indiquer que les futurs enseignants d'origine immigrée qu'il a pu interroger n'ont pas des caractéristiques sociales foncièrement différentes de 
celles de l'ensemble des immigrés. Ceci suggère plusieurs résultats importants qui mériteraient d'être approfondis.

En premier lieu, et contrairement au monde de l'entreprise étudié dans la plupart des autres contributions, le monde de l'enseignement primaire et secondaire ne se signale pas par un degré significatif de comportements discriminatoires. Pour peu que la mobilisation individuelle et familiale atteigne un certain seuil, la profession d'enseignant du primaire ou du secondaire reste accessible aux enfants de l'immigration et il pourrait se faire qu'il en soit ainsi de façon générale pour la fonction publique, qui jouerait alors un rôle de premier plan dans l'intégration des secondes générations. Cette impression reste évidemment à confirmer par des enquêtes plus générales.

En second lieu, la comparaison avec la Suisse pourrait bien illustrer l'effet différenciateur et, pour tout dire, discriminateur, d'un droit de la nationalité restrictif, qui réserve la naturalisation à une petite minorité d'immigrés au lieu de la rendre accessible, comme c'est le cas de la France, à la grande majorité des enfants d'immigrés nés en France ou socialisés en France. Une lecture optimiste mais sans doute raisonnable de l'article de Frédéric Charles suggère que le tiercé gagnant pour favoriser l'intégration professionnelle de la seconde génération réunit en fin de compte un droit de la nationalité accueillant, une fonction publique respectueuse des principes d'ouverture et de neutralité, et des familles immigrées fortement mobilisées sur un projet éducatif en dépit de leurs handicaps culturels.

Le point de départ de l'article de Marnia Belhadj est une interrogation originale: dans quelle mesure les travailleurs sociaux qui œuvrent à l'intégration des quartiers où se concentre une part importante des immigrés sont-ils eux-mêmes issus de l'immigration? Sont-ils en continuité ou en rupture avec leur milieu d'origine ? La même question se posait dans les générations précédentes pour les milieux populaires qui avaient précédé l'immigration et qui étaient souvent issus de l'exode rural. Le parallélisme est exploré à travers une vingtaine d'entretiens avec des travailleurs sociaux d'origine immigrée.

Indéniablement, la familiarité des éducateurs avec le milieu de l'immigration est perçue comme un atout, de même que l'expérience associative ou militante; elle permettrait même de rompre avec la distance bureaucratique que pratiquaient les services sociaux d'antan. Comme, par ailleurs, le niveau de la formation universitaire reçue s'est nettement élevé en l'espace d'une ou deux générations, la qualité du travail social effectué n'a pu que s'améliorer. Mais les contenus de l'action sociale ont changé : davantage centrés sur l'insertion socio-professionnelle que sur l'aide aux familles, plus étroitement liés aux demandes des élus locaux et aux politiques de la ville.

Se pose alors la question de savoir si le recrutement de ces nouveaux travailleurs sociaux se fait explicitement en fonction de leurs origines (auquel cas on pourrait parler d'une sélection « ethnique ») ou si le critère d'une familiarité acquise de longue date s'interprète simplement comme une connaissance du terrain, une sorte de " compétence territoriale » qui préserve les formes républicaines du recrutement. La formation reçue a beau inclure des enseignements sur les relations inter-ethniques ou la sociologie de l'immigration, le travail de terrain a beau mobiliser des compétences de médiation et de conciliation impliquant une connaissance intime des façons de faire, cela ne prépare pas à traiter l'ensemble des problèmes culturels que présente une immigration aux provenances multiples. Surtout, les directeurs de structure qui recrutent des travailleurs sociaux éprouvent aussi la nécessité d'établir la bonne distance avec le milieu d'origine et de neutraliser les préférences ou les répulsions culturelles héritées du passé.

À ce stade, les propos rapportés par Marnia Belhadj ne laissent pas d'être ambigus : il faudrait préserver une certaine "diversité » du recrutement. Mais les exemples britannique et américain montrent bien que la diversité peut aboutir paradoxalement à l'uniformité s'il s'agit d'offrir à chaque groupe ethnique le travailleur social, le vendeur ou l'élu qui appartiendra au même groupe que lui : l'appariement du même avec le même qui devrait s'établir au nom de la relation de confiance ou du droit à la représentation aboutit alors au contraire de la diversité. La volonté de n'avoir affaire qu'avec son semblable peut alors, pour le coup, tourner à la discrimination. La pratique française dit l'inverse : je dois être capable, en tant que patient, consommateur ou électeur, de m'accommoder d'un médecin, d'une aide-soignante, d'un éducateur, d'un vendeur ou d'un député qui puisse avoir de toutes autres origines que les miennes. La « diversité républicaine », si on ose la désigner ainsi, consiste précisément dans ce brassage. Marnia 
Belhadj n'entre pas dans ce débat, qui la mènerait trop loin, mais on soupçonne qu'il figure à l'arrièreplan des tensions ou des oscillations qu'elle décrit : d'un côté, le désir d'entretenir des relations de confiance avec les jeunes et les familles, qui peut aller jusqu'au soutien psychologique et au partage des mêmes valeurs culturelles; de l'autre, la volonté de fournir une aide à l'insertion sur un mode plus professionnel et plus distant.

Avec le travail de Didier Gélot et Claude Minni, qui clôt ce recueil, nous restons en apparence sur une question sectorielle, l'accès des immigrés à la formation professionnelle continue. En réalité, l'angle de vue s'élargit considérablement. Non seulement, l'analyse brasse toutes les enquêtes nationales disponibles en matière d'emploi et de formation, mais elle pose la question de savoir comment se situent les disparités entre immigrés et non-immigrés par rapport à l'ensemble des inégalités qui traversent la société française. Gélot et Minni commencent par montrer l'ampleur du problème : les immigrés recourent environ deux fois moins que le reste des actifs à la formation continue. Cet écart est si fort qu'il persiste une fois qu'on a neutralisé l'effet de la taille d'établissement et du secteur d'activité : on l'observe encore au sein des petites entreprises du bâtiment. La formation continue ne peut donc jouer le rôle de seconde chance qu'elle est censée jouer pour les actifs qui ont dû interrompre de façon précoce leur scolarité. Elle contribue même à creuser les écarts initiaux de formation. Mais ce paradoxe, on le sait, caractérise aussi l'ensemble de la formation professionnelle continue, indépendamment de l'immigration. Du coup, le problème se pose pour toutes les catégories d'immigrés, quelles que soient leurs origines nationales, en fort contraste avec l'accès à l'emploi, qui pénalise fortement l'origine maghrébine.

Un autre élément majeur à prendre en compte, selon les auteurs, est le fait que les immigrés sont tout autant, sinon davantage, demandeurs de formation que les Français. Les moins qualifiés d'entre eux déclarent plus fréquemment des besoins de formation non satisfaits. Mais cette demande s'inscrit dans le temps immédiat. Quand on en vient aux demandes de formation pour l'avenir, liées aux projets de mobilité professionnelle, ce sont les Français qui deviennent plus demandeurs de formation que les immigrés, comme s'ils anticipaient davantage un accès plus aisé à la mobilité. L'écart semble toutefois se réduire quand on passe des immigrés récents aux immigrés installés de plus longue date.

Conscients de la complexité des facteurs qui s'enchevêtrent pour expliquer l'ampleur des besoins non exprimés et des demandes expresses, Gélot et Minni s'interrogent sur les orientations actuelles de l'action publique dans ce domaine. La loi de 2004 sur la formation professionnelle, qui a renouvelé la loi fondatrice de 1971, n'a pas cherché à cibler la population active immigrée. Rien ne permet de penser, dans ces conditions, que l'application du simple droit commun permettra de combler l'écart que la mise en œuvre de la formation continue n'a cessé de creuser entre les divers groupes, et spécialement entre les natifs et les immigrés. Faudra-t-il se tourner vers une politique de compensation active pour attaquer sérieusement le problème? Le refus dogmatique des actions compensatrices ciblées n'est sans doute pas une bonne solution. Mais avant de trancher la question ici, il faudrait sans doute en savoir davantage sur les obstacles qui entravent l'expression des besoins et la concrétisation des offres de formation: obstacles linguistiques? Obstacles liés à l'organisation horaire du travail ? Report des ambitions sur la génération suivante?

Là encore, les auteurs demeurent prudents sur la question de la discrimination, qu'ils n'invoquent pas comme facteur principal d'une exclusion des immigrés vis-àvis de la formation continue. C'est sans doute l'une des principales difficultés des travaux de recherche sur le sort des immigrés que de devoir prendre ainsi en considération la combinaison de deux dimensions: d'un côté, l'existence de comportements discriminatoires qui prennent pour cible certains groupes d'immigrés et qui sont parfaitement attestés par les tests en situation et les analyses «toutes choses égales d'ailleurs »; de l'autre, la persistance des inégalités sociales dans la société où les immigrés doivent tant bien que mal s'insérer, ce qui les amène à partager les conditions de vie difficiles d'une partie des milieux populaires. Les deux phénomènes se cumulent mais ne se confondent pas. 